\title{
Equilibrium delay and non-existence of equilibrium in unanimity bargaining games
}

Citation for published version (APA):

Britz, V., Herings, P. J. J., \& Predtetchinski, A. (2014). Equilibrium delay and non-existence of equilibrium in unanimity bargaining games. Maastricht University, Graduate School of Business and Economics. GSBE Research Memoranda No. 019 https://doi.org/10.26481/umagsb.2014019

Document status and date:

Published: 01/01/2014

DOI:

10.26481/umagsb.2014019

Document Version:

Publisher's PDF, also known as Version of record

\section{Please check the document version of this publication:}

- A submitted manuscript is the version of the article upon submission and before peer-review. There can be important differences between the submitted version and the official published version of record.

People interested in the research are advised to contact the author for the final version of the publication, or visit the DOI to the publisher's website.

- The final author version and the galley proof are versions of the publication after peer review.

- The final published version features the final layout of the paper including the volume, issue and page numbers.

Link to publication

\footnotetext{
General rights rights.

- You may freely distribute the URL identifying the publication in the public portal. please follow below link for the End User Agreement:

www.umlib.nl/taverne-license

Take down policy

If you believe that this document breaches copyright please contact us at:

repository@maastrichtuniversity.nl

providing details and we will investigate your claim.
}

Copyright and moral rights for the publications made accessible in the public portal are retained by the authors and/or other copyright owners and it is a condition of accessing publications that users recognise and abide by the legal requirements associated with these

- Users may download and print one copy of any publication from the public portal for the purpose of private study or research.

- You may not further distribute the material or use it for any profit-making activity or commercial gain

If the publication is distributed under the terms of Article $25 \mathrm{fa}$ of the Dutch Copyright Act, indicated by the "Taverne" license above, 
Volker Britz,

P. Jean-Jacques Herings,

Arkadi Predtetchinski

Equilibrium Delay and Nonexistence of Equilibrium in Unanimity Bargaining Games

$\mathrm{RM} / 14 / 019$

\section{GSBE}

Maastricht University School of Business and Economics

Graduate School of Business and Economics

P.O Box 616

NL- 6200 MD Maastricht

The Netherlands 


\title{
Equilibrium Delay and Non-existence of Equilibrium in Unanimity Bargaining Games
}

\author{
Volker Britz $^{1} \quad$ P. Jean-Jacques Herings ${ }^{2} \quad$ Arkadi Predtetchinski ${ }^{3}$
}

May 13, 2014

${ }^{1}$ V. Britz (vbritz@ethz.ch), Center of Economic Research at ETH Zurich (CER-ETH).

${ }^{2}$ P.J.J. Herings (p.herings@maastrichtuniversity.nl), Department of Economics, Maastricht University.

${ }^{3}$ A. Predtetchinski (a.predtetchinski@maastrichtuniversity.nl), Department of Economics, Maastricht University. 


\begin{abstract}
We consider a class of perfect information unanimity bargaining games, where the players have to choose a payoff vector from a fixed set of feasible payoffs. The proposer and the order of the responding players is determined by a state that evolves stochastically over time. The probability distribution of the state in the next period is determined jointly by the current state and the identity of the player who rejects the current proposal. This protocol encompasses a vast number of special cases studied in the literature. These special cases have in common that equilibria in pure stationary strategies exist, are efficient, are characterized by the absence of delay, and converge to a unique limit corresponding to an asymmetric Nash bargaining solution. For our more general protocol, we show that subgame perfect equilibria in pure stationary strategies need not exist. When such equilibria do exist, they may exhibit delay. Limit equilibria as the players become infinitely patient need not be unique.
\end{abstract}

Keywords: Strategic Bargaining, Subgame Perfect Equilibrium, Stationary Strategies, Nash Bargaining Solution.

JEL CODES: C78. 


\section{Introduction}

In his seminal paper, Rubinstein (1982) studies the division of a surplus among two impatient players through a non-cooperative bargaining game. Following this contribution, a rich literature has emerged which extends and generalizes Rubinstein's approach. In this paper, we point out a number of general results which have persistently and recurrently emerged from this literature, and we explore the boundaries of their scope. More in particular, we give some examples where a further generalization of the model leads to a break-down of these results. While the analysis of unanimity bargaining games has typically focussed on subgame perfect equilibria in pure stationary strategies, we show that in a more general set-up such equilibria need not exist.

For the purpose of this paper, we mean by a unanimity bargaining game a noncooperative game with the following characteristics. There is a finite number of players who need to make a unanimous choice for one particular payoff vector within a fixed set of feasible payoffs. The game is set in discrete time. In each round of the game, one player is the proposer. His role is to suggest one particular feasible payoff vector. The other players then sequentially accept or reject this proposal in some fixed order. If all players agree to the proposal, the game ends and the agreed payoffs are realized. As soon as one of the players rejects the current proposal, the game proceeds to the next round. The time horizon is infinite, so players may disagree forever, which yields a payoff of zero to every player. The payoffs are subject to time-discounting. When an agreement is reached, the payoff of each player is multiplied by an exogenously given discount factor for each round which has previously passed without agreement. ${ }^{1}$

In order to complete the description of a unanimity bargaining game, one has to specify a rule which determines who is the proposer in each round. We will refer to this rule as the protocol in the sequel. Rubinstein (1982) studies a game with only two players who simply take turns in making proposals, the alternating offer protocol. Rubinstein finds a unique subgame perfect equilibrium. The equilibrium strategies happen to be stationary. It is well-known that the uniqueness of subgame perfect equilibrium breaks down in unanimity bargaining games with more than two players. With regard to those games, the literature focusses on subgame perfect equilibria in stationary strategies, which allow sharp

\footnotetext{
${ }^{1}$ Instead of time-discounting, some authors assume an exogenous breakdown of the negotiation to occur after each disagreement with probability $1-\delta$. Time-discounting and the possibility of an exogenous breakdown are largely interchangeable interpretations of $\delta$. The term "bargaining friction" can be used to capture both of them. The importance of the bargaining friction lies in the fact that it creates an incentive to come to an agreement sooner rather than later.
} 
predictions of the equilibrium payoffs at least when the discount factor is sufficiently close to one. Arguably the most obvious generalization of Rubinstein's alternating offer protocol to the case with more than two players is the rotating protocol, under which players become proposers in ascending order, and player one proposes again after player $n$. One alternative proper generalization of the alternating offers protocol is the rejector-proposes protocol. Under that rule, the first player to reject the current proposal becomes the next proposer. The rejector-proposes protocol is an example of an endogenous protocol in which the actions taken by the players throughout the game have an influence on the proposer selection. One important example of a protocol that is not a proper generalization of Rubinstein's alternating offers is the time-invariant probability protocol. That protocol consists of an exogenously given probability distribution from which the proposer is drawn in each round.

The literature on unanimity bargaining games has established some results that are generally valid no matter which of these protocols is assumed.

1. Subgame perfect equilibria in pure stationary strategies exist and they are efficient.

2. Subgame perfect equilibria in pure stationary strategies predict immediate agreement in every subgame.

3. In the limit as $\delta$ approaches one, all the proposals of all the stationary subgame perfect equilibria converge to a unique limit proposal.

4. The limit proposal is equal to an asymmetric Nash Bargaining Solution.

The limit proposal itself depends on the distribution of bargaining power inherent in the protocol. Kultti and Vartiainen (2010) show that the limit proposal is the Nash Bargaining Solution under the rotating protocol. Miyakawa (2008) and Laruelle and Valenciano (2008) study the time-invariant probability protocol. In this case, the limit proposal corresponds to the asymmetric Nash Bargaining Solution, where the vector of bargaining weights is given by the time-invariant probability distribution. Britz, Herings, and Predtetchinski (2010) study a protocol where the proposer is chosen by a Markov process. That is, there are $n$ probability distributions on the $n$ players. The identity of the proposer in the current round determines which of the $n$ probability distributions is used to draw the proposer in the following round. The Markov process is assumed to have a stationary distribution. Then, the limit proposal corresponds to an asymmetric Nash Bargaining Solution, where the vector of bargaining weights is given by the stationary distribution of the Markov process. This Markovian protocol is a generalization of the time-invariant probabilities 
and the rotating protocols - in fact, it seems to be the most general among the exogenous protocols.

Britz, Herings, and Predtetchinski (2012) complement that analysis with a study of endogenous protocols. More in particular, they consider a protocol that consists of $n$ probability distributions on the $n$ players and the identity of the player who rejects the current proposal determines which one of those probability distributions will be used to draw the following proposer. It turns out that the limit proposal depends only on the probabilities with which each player becomes the proposer after his own rejection. More in particular, the limit proposal is again an asymmetric Nash Bargaining Solution where the vector of bargaining weights is proportional to the vector of probabilities with which the players propose after their own rejections. ${ }^{2}$ One implication of this result is that the limit proposal corresponds to the Nash Bargaining Solution under the rejector-proposes protocol.

The plan of the paper is as follows. We start by formally describing a unanimity bargaining game with a quite general protocol in Section 2. In particular, this protocol is designed such that it includes all the aforementioned protocols as special cases. Section 3 summarizes the results in the existing literature regarding existence, efficiency, immediate agreement, uniqueness, and characterization of equilibrium. Within the framework of that model, we will give some examples to demonstrate that such results are not valid for more general protocols. Section 4 presents an example where pure stationary subgame perfect equilibrium predicts delay, inefficiency, and non-uniqueness of limit equilibrium. Section 5 shows the example to be robust. Herings and Predtetchinski (2009) have shown for the protocol with time-invariant recognition probabilities that pure subgame perfect equilibria in stationary strategies exist even when the set of feasible payoffs is non-convex. We show in Section 6 that even when the set of feasible payoffs corresponds to the division of a surplus, a pure subgame perfect equilibrium in stationary strategies may not exist for general protocols.

Our results complement some of the examples of equilibrium delay and non-existence found in the literature. An example of a stationary subgame perfect equilibrium exhibiting delay has been given in Chatterjee, Dutta, Ray, and Sengupta (1993) in the context of coalitional bargaining. Unlike the unanimity bargaining games considered here, in coalitional bargaining games a proposing player may choose to make an offer to a subset of the players. The approval of the proposal by all players in the chosen coalition is then

\footnotetext{
${ }^{2}$ This result requires, however, that these probabilities to propose after one's own rejection are strictly positive for all players.
} 
sufficient for the proposal to pass. Also in a coalitional bargaining context, Bloch (1996) shows that subgame perfect equilibria in pure stationary strategies need not exist. Merlo and Wilson (1995) show that delay in a stationary subgame perfect equilibrium is possible if the size of the cake changes stochastically over time. Jéhiel and Moldovanu (1995) show that delay can arise due to externalities. In addition to these examples where delay arises in a complete and perfect information framework, there is a literature on bargaining delays when the parties are asymmetrically informed, see e.g. the review in Ausubel, Cramton, and Deneckere (2002).

\section{Model}

We consider a non-cooperative bargaining game $G\left(N, V, S, \iota, p^{0}, p, \delta\right)$, where $N=$ $\{1, \ldots, n\}$ is the set of players and $V \subset \mathbb{R}_{+}^{n}$ is the set of feasible payoff allocations. Bargaining takes place in discrete time $t=0,1, \ldots$ In each round one player is selected as a proposer, and proposes an element $v$ of $V$. Next the players sequentially respond to the proposal and in case of unanimous acceptance, the proposal is implemented and the game ends with payoffs $v$ to the players. As soon as one player rejects, the game breaks down with probability $1-\delta$, and continues to the next round with probability $\delta$. In case of breakdown, as well as in case of perpetual disagreement, payoffs to all players are equal to zero.

Our emphasis will be on the role of the bargaining procedure in determining the bargaining outcome. The set of feasible payoffs $V$ is therefore kept fixed in each round, but our bargaining procedure is allowed to be quite general. To achieve this, we make use of a finite state space $S$. The function $\iota: S \rightarrow N \times \Pi$, where $\Pi$ is the set of permutations on $N$, assigns to each state a proposer and an order of responders. That is, if $\iota(s)=(i, \pi)$, then Player $i$ is the proposer in state $s$ and all players sequentially respond to the proposal in the order $\pi(1), \ldots, \pi(n)$ given by the permutation $\pi$. In round $t=0$, the initial state is determined by the probability distribution $p^{0} \in \Delta(S)$, where $\Delta(S)$ is the set of probability distributions on $S$. In any round $t>0$, the state of the game is determined by transition functions $p^{j}: S \rightarrow \Delta(S)$, one for each Player $j \in N$. If Player $j \in N$ rejects the proposal at time $t$ when the game is in state $s$, then $p^{j}(s)$ returns the probability distribution from which the state at time $t+1$ is drawn conditional on the continuation of the negotiations.

Many protocols that have been studied in the bargaining literature are special cases of the class of protocols described above, up to relatively unimportant modeling details. Such modeling details concern whether there is some probability of breakdown of negotiations, or 
whether players have time preferences. In case time preferences take the discounted utility form, although conceptually different from the risk preferences that are needed to study models with breakdown, both approaches lead to the same results as argued in Binmore, Rubinstein, and Wolinsky (1986). Another issue is whether players vote simultaneously or sequentially. Under simultaneous voting, the solution concept of subgame perfection has less bite, and on top of subgame perfection it is typically required that players use stage-undominated voting strategies to avoid coordination problems. We study sequential voting in this paper.

The protocol studied in the seminal contribution of Rubinstein (1982) corresponds to the case where $S=N=\{1,2\}$, Player $s$ is the proposer in state $s$, and state transitions are such that the state alternates between periods. Player 1 is the initial proposer. Formally, in Rubinstein (1982), Player $s$ does not vote on his own proposal in state $s$, but allowing for this option would not change the analysis. Kultti and Vartiainen (2010) consider a multilateral extension of alternating offer bargaining, where proposer rotate in making offers. Their model corresponds to the case where $S=N$, Player $s$ is the proposer in state $s$, and the state transition is to state $s+1$ modulo $n$ with probability 1 if the current state is $s$. Banks and Duggan (2000) study legislative bargaining models and consider time-invariant recognition probabilities in a framework incorporating unanimity rule as a special case. Time-invariant recognition probabilities result when $S=N$ and there is a fixed probability distribution $p^{0}$ on $S$ such that the proposer is selected in accordance with $p^{0}$ in every time period. Kalandrakis (2004) and Britz, Herings, and Predtetchinski (2010) consider the case where $S=N$ and require that for all $j, k \in N, p^{j}=p^{k}$. For $s \in S$, it holds that $\iota(s)=\left(s, \pi^{0}\right)$, where $\pi^{0}$ is the identity. The state denotes the current proposer and Player $i$ responds before Player $j$ if and only if $i<j$. State transitions are not influenced by the identity of the rejecting player. Merlo and Wilson (1995) also assume that for all $j, k \in N, p^{j}=p^{k}$, and even allow for an infinite state space $S$. They also allow the set of feasible payoffs to depend on the state $s$, but since our attention here is on the influence of the protocol on the allocation of the payoffs, we consider a fixed set $V$ instead.

All the bargaining protocols described in the previous paragraph have in common that the actions taken by the players are without consequence for the way the bargaining protocol proceeds in case of a rejection, i.e. for all $j, k \in N$ it holds that $p^{j}=p^{k}$. We refer to these protocols as exogenous.

The rejector-becomes-proposer protocol is introduced in Selten (1981) in a coalitional bargaining set-up and specifies that the player who rejects the current proposal is automatically called upon to make the next proposal. Kawamori (2008) generalizes this protocol to allow for a general probabilistic selection of a new proposer, conditional on who rejects 
the current proposal. When we apply his coalitional bargaining model to our unanimity bargaining set-up, we obtain the case where $S=N$, for $s \in S$ it holds that $\iota(s)=\left(s, \pi^{0}\right)$, where $\pi^{0}$ is some fixed permutation of the players, and for all $s, s^{\prime} \in S, p^{j}(s)=p^{j}\left(s^{\prime}\right)$.

Whenever for some $j, k \in N, p^{j} \neq p^{k}$, the actions of the players influence the way the bargaining protocol proceeds, and we refer to such protocols as endogenous protocols. The rejector-becomes-proposer protocol is a key example of an endogenous protocol.

\section{Results in the Existing Literature}

Multilateral bargaining games are known to admit a wide multiplicity of subgame perfect equilibria, see Herrero (1985) and Haller (1986). It is therefore common in the literature to restrict attention to subgame perfect equilibria in stationary strategies. Although ideally the notion of stationarity should follow endogenously from the specification of the game as in Maskin and Tirole (2001), the literature typically takes the more ad hoc approach described below, which in general leads to weaker notions of stationary equilibrium than the one of Maskin and Tirole (2001).

A stationary strategy for Player $i$ in the game $G(\delta)$ specifies, for each state $s \in S$ such that $\iota(s)=(i, \pi)$ for some $\pi \in \Pi$, a proposal $\theta^{s} \in V$, and for each state $s \in S$ an acceptance set $A^{i, s} \subset V$. A stationary strategy of a player specifies a unique action for each of his decision nodes. This action depends only on the state and not on any other aspect of the history if the player is a proposer and on the state as well as the proposal made if the player is a responder. A stationary strategy profile $(\theta, A)$ leads to a unique probability distribution over payoffs in $V$, so determines the utility $u_{i}(\theta, A)$ of Player $i \in N$. Conditional utilities are denoted by $u_{i}(\theta, A \mid s)$. The social acceptance set in state $s \in S$ is defined as $A^{s}=\cap_{i \in N} A^{i, s}$. The social acceptance set consists of all alternatives that are unanimously accepted when proposed in state $s$.

Definition 3.1 A subgame perfect equilibrium in stationary strategies (SSPE) is a profile of stationary strategies which is a subgame perfect equilibrium of the game.

We make the following standard assumptions on $V$, where we use the notation $V_{+}=$ $V \cap \mathbb{R}_{+}^{n}$ and $\partial V_{+}$is the set of weakly Pareto efficient points in $V_{+}$. Moreover, a vector $\eta$ with $\|\eta\|=1$ is said to be normal to the set $V$ at a point $\bar{v} \in V$ if $(v-\bar{v})^{\top} \eta \leq 0$ for every $v \in V$. The set of all vectors $\eta$ normal to $V$ at $\bar{v}$ is called the normal to $V$ at $\bar{v}$.

Assumption A The set $V$ is closed, convex, and comprehensive from below. The origin 
lies in the interior of $V$. The set $V_{+}$is bounded and all points in $\partial V_{+}$are strongly Pareto efficient. There is a unique vector in the normal to $V$ at every $v \in \partial V_{+}$.

A stationary strategy profile $(\theta, A)$ is said to have no delay if for every $s \in S$ it holds that $\theta^{s} \in A^{s}$. A stationary strategy profile $(\theta, A)$ is said to be efficient if for every $s \in S$ it holds that $\theta^{s} \in \partial V_{+}$.

Apart from the analysis of $G(\delta)$, the literature also typically studies the behavior of equilibria when the continuation probability $\delta$ tends to 1 .

Definition 3.2 The profile of proposals $\bar{\theta}=\left(\bar{\theta}^{s}\right)_{s \in S}$ is a limit equilibrium if there is a sequence $\left\{\delta_{m}\right\}_{m \in \mathbb{N}}$ of continuation probabilities in $[0,1)$ converging to 1 and a sequence of profiles $\left\{\theta\left(\delta_{m}\right)\right\}_{m \in \mathbb{N}}=\left\{\left(\theta^{s}\left(\delta_{m}\right)_{s \in S}\right)\right\}_{m \in \mathbb{N}}$, where $\theta\left(\delta_{m}\right)$ is an SSPE profile of proposals of the game $G\left(\delta_{m}\right)$, such that $\lim _{m \rightarrow \infty} \theta\left(\delta_{m}\right)=\bar{\theta}$.

A limit equilibrium is a profile of proposals that can be approximated arbitrarily close by an SSPE profile of proposals when the probability of breakdown is sufficiently small. Of particular interest is the relationship between limit equilibria and the asymmetric Nash bargaining solution with positive weights $\mu \in \mathbb{R}_{+}^{n} \backslash\{0\}$, denoted $\mu$-ANBS and defined as follows.

Definition 3.3 The asymmetric Nash product with weights $\mu \in \mathbb{R}_{+}^{n} \backslash\{0\}$ is the function $f: V_{+} \rightarrow \mathbb{R}$ defined by

$$
f(v)=\prod_{i \in N}\left(v_{i}\right)^{\mu_{i}}
$$

The $\mu$-ANBS is the unique maximizer of the function $f$ on the set $V_{+}$.

Britz, Herings, and Predtetchinski (2010) study the class of exogenous protocols characterized by the following assumption.

Assumption B It holds that $S=N$, for every $s \in S, \iota(s)=\left(s, \pi^{0}\right)$ with $\pi^{0}$ the identity, for all $j, k \in N, p^{j}=p^{k}$, and the matrix $M=\left[p^{j}(1), \ldots, p^{j}(n)\right]$ is irreducible.

An irreducible matrix $M$ has a unique stationary distribution $\mu$. Recall that a stationary distribution $\mu$ is a probability distribution on the set of states satisfying $M \mu=\mu$. The class of protocols satisfying Assumption B is sufficiently rich to encompass alternating and rotating offers and time-invariant recognition probabilities.

Britz, Herings, and Predtetchinski (2012) study the class of endogenous protocols characterized by the following assumption. 
Assumption C It holds that $S=N \times \Pi$, for every $s \in S, \iota(s)=s$, for every $j \in N$, for all $s, s^{\prime} \in S, p^{j}(s)=p^{j}\left(s^{\prime}\right)$. There exists $(j, \pi) \in S$ such that $p^{j}(s)$ assigns positive probability to $(j, \pi)$.

We associate to each protocol satisfying Assumption $C$ the weights $\mu>0$ given by $\mu_{j}=\sum_{(j, \pi) \in S} p_{(j, \pi)}^{j}(s)$, where the choice of $s$ is irrelevant by Assumption C, so $\mu_{j}$ is the probability that Player $j$ becomes the next proposer conditional on a rejection by himself. The class of protocols satisfying Assumption C is sufficiently rich to include the rejectorbecomes-proposer protocol as well as the generalization by Kawamori (2008).

The following result follows from Britz, Herings, and Predtetchinski (2010, 2012).

Theorem 3.4 If Assumptions $A$ and $B$, or Assumptions $A$ and $C$, are satisfied then

1. An SSPE exists.

2. Every SSPE has no delay.

3. Every SSPE is efficient.

4. There is a unique limit equilibrium.

5. All limit equilibrium proposals are equal to the $\mu$-ANBS.

The five claims in Theorem 3.4 can be thought of as increasingly demanding. The characterization of limit equilibrium proposals as a weighted Nash bargaining solution has been shown in Binmore, Rubinstein, and Wolinsky (1986) for bilateral bargaining. For multilateral bargaining this result is obtained in Hart and Mas-Colell (1996) for uniform time-invariant recognition probabilities, in Miyakawa (2008) and Laruelle and Valenciano (2008) for general time-invariant recognition probabilities, and in Kultti and Vartiainen (2010) for rotating offers. Theorem 3.4 includes these results as special cases. The five claims of Theorem 3.4 and in particular the limit equilibrium payoffs are independent of the order in which the responding players accept or rejects the proposal. This is noteworthy in the case of an endogenous protocol as in Britz, Herings, and Predtetchinski (2012) - for instance, one might have conjectured that the rejector-proposes protocol favors the player who comes first in the responder order. 


\section{Failure of No Delay, Efficiency, and Uniqueness}

Ideally one would like to prove Theorem 3.4 for the entire class of bargaining protocols as laid down in Section 2, up to standard regularity assumptions. In this section we present an example where properties (2)-(5) of Theorem 3.4 are violated. Moreover, the example is minimal in the sense that it has $S=N=\{1,2,3\}$, and for two states out of three we have $p^{j}(s)=p^{k}(s)$, for all $j, k \in N$. The protocol is therefore exogenous and Assumption $\mathrm{B}$ is satisfied, with the exception of one state.

Example 4.1 There are three players and three states, $S=N=\{1,2,3\}$. Player $i$ is the proposer in state $i$, and players respond in ascending order, so we have

$$
\begin{aligned}
& \iota(1)=\left(1, \pi^{0}\right), \\
& \iota(2)=\left(2, \pi^{0}\right), \\
& \iota(3)=\left(3, \pi^{0}\right),
\end{aligned}
$$

where $\pi^{0}$ is the identity. Players have to divide a surplus of one unit, $V=\left\{v \in \mathbb{R}^{3} \mid v_{1}+\right.$ $\left.v_{2}+v_{3} \leq 1\right\}$. This set clearly satisfies Assumption A. In state $s=1$, the transitions depend on the identity of the player who rejects a proposal. If Player 1 rejects a proposal, each player becomes the next proposer with equal probability. If Player 2 or Player 3 rejects a proposal, this player becomes the next proposer with probability one. We have

$$
\begin{aligned}
& p^{1}(1)=\left(\frac{1}{3}, \frac{1}{3}, \frac{1}{3}\right), \\
& p^{2}(1)=(0,1,0), \\
& p^{3}(1)=(0,0,1) .
\end{aligned}
$$

In states $s=2,3$, the transitions are independent of the identity of the rejecting player. Once in state 2, Player 2 remains the proposer forever. The same holds true in state 3 . We have

$$
\begin{aligned}
& p^{i}(2)=(0,1,0), \quad i \in N \\
& p^{i}(3)=(0,0,1), \quad i \in N .
\end{aligned}
$$

The next result claims not only that equilibria may exhibit delay, but even makes the stronger statement that all SSPEs feature delay.

Proposition 4.2 For $\delta>1 / 2$, every SSPE in Example 4.1 has delay. 
Proof. Suppose $(\theta, A)$ is an SSPE which has no delay. Consider a subgame starting with a proposal by Player 2 in state 2 . In this subgame, Player 2 remains the proposer forever, and it is straightforward to verify that the subgame has a unique SSPE where Player 2 captures the entire surplus. It holds that $u(\theta, A \mid 2)=(0,1,0)$. By a completely symmetric argument, we find that $u(\theta, A \mid 3)=(0,0,1)$. Now consider a subgame starting with a proposal by Player 1 in state 1 . Since $(\theta, A)$ has no delay, Player 2 accepts $\theta^{1}$ and it holds that $\theta_{2}^{1} \geq \delta$, since a rejection by Player 2 leads to a breakdown with probability $\delta$ and a transition to state 2 and a payoff of 1 for Player 2 with probability $1-\delta$. Similarly, it holds that $\theta_{3}^{1} \geq \delta$, since a rejection by Player 3 leads to a breakdown with probability $\delta$ and a transition to state 3 and a payoff of 1 for Player 3 with probability $1-\delta$. It follows that

$$
\theta_{1}^{1} \leq 1-\theta_{2}^{1}-\theta_{3}^{1} \leq 1-2 \delta<0
$$

Since Player 1 can ensure a non-negative payoff by a strategy that rejects all proposals, we have obtained a contradiction to $(\theta, A)$ being an SSPE which has no delay.

The intuition behind the example is the following. If Player 2 rejects the proposal of Player 1 , then the game goes to an absorbing state where Player 2 remains the proposer forever. It is well-known that in any SSPE of such a subgame Player 2 would capture the entire surplus. Thus, when the game is in state 1 Player 2 can guarantee himself a payoff of $\delta$ by rejecting Player 1's proposal. In any SSPE with no delay, Player 1 would need to offer at least the amount $\delta$ to Player 2. The same argument applies to Player 3: If Player 3 rejects a proposal of Player 1, the game goes to an absorbing state where Player 3 remains the proposer forever and can capture the entire surplus. Thus, when Player 3 reacts to the proposal of Player 1, he will not accept any less than $\delta$. Indeed, the sum of the responding players' reservation payoffs is equal to $2 \delta$. We can see that if $\delta>\frac{1}{2}$, then the available surplus is not sufficient for Player 1 to pay the other two players their reservation payoffs. Consequently, if $\delta>\frac{1}{2}$, no agreement can be reached in state 1 .

In the example, states 2 and 3 are absorbing. However, small changes in all transition probabilities do not affect the main argument, and would still lead to the conclusion that for a sufficiently high value of the continuation probability $\delta$, all SSPEs have delay. Section 5 studies the case where transition probabilities are perturbed in more detail.

The next issue is whether there is an SSPE with delay in Example 4.1. Consider a strategy profile $(\bar{\theta}, \bar{A})$ with proposals $\bar{\theta}^{1} \in V, \bar{\theta}^{2}=(0,1,0)$, and $\bar{\theta}^{3}=(0,0,1)$, and 
acceptance sets

$$
\begin{array}{rlr}
\bar{A}^{1, s} & =\left\{v \in V \mid v_{1} \geq 0\right\}, & \\
\bar{A}^{2,1} & =\left\{v \in V \mid v_{2} \geq \delta, v_{3} \geq \delta\right\}, & \\
\bar{A}^{2,2} & =\left\{v \in V \mid v_{2} \geq \delta\right\}, & \\
\bar{A}^{2,3} & =\left\{v \in V \mid v_{2} \geq 0\right\}, & \\
\bar{A}^{3, s} & =\left\{v \in V \mid v_{3} \geq \delta\right\}, & \\
\bar{A}^{3,2} & =\left\{v \in V \mid v_{3} \geq 0\right\} . &
\end{array}
$$

When players play according to $(\bar{\theta}, \bar{A})$, Player 1 makes a particular proposal belonging to $V$ in state 1 , which will be rejected by some player when $\delta>1 / 2$. More precisely, the proposal $\bar{\theta}^{1}$ in state 1 is rejected by Player 1 when $\bar{\theta}_{1}^{1}<0$ and is rejected by Player 2 otherwise. Notice that in state 1, Player 2 would even reject the counterfactual proposal $(0,1,0)$, since acceptance of such a proposal would lead to a rejection by Player 3 , followed by breakdown of the negotiations or a transition to state 3 .

If $\bar{\theta}_{1}^{1}<0$, after the rejection by Player 1 , negotiations break down with probability $1-\delta$ and continue with probability $\delta$. If negotiations continue, transitions occur with equal probability to each of the three states, a rejection of proposal $\bar{\theta}^{1}$ by Player 1 in state 1 , an acceptance of payoff vector $(0,1,0)$ in state 2 , and an acceptance of payoff vector $(0,0,1)$ in state 3.

If $\bar{\theta}_{1}^{1} \geq 0$, after the rejection by Player 2 , negotiations break down with probability $1-\delta$ and continue in state 2 with probability $\delta$. In the latter case, the payoff vector $(0,1,0)$ is proposed and accepted.

Proposition 4.3 For $\delta>1 / 2$, the strategy profile $(\bar{\theta}, \bar{A})$ is an SSPE in Example 4.1.

Proof. To show that $(\bar{\theta}, \bar{A})$ is an SSPE, it suffices to verify the one-shot deviation property, see for instance Fudenberg and Tirole (1991). We consider three cases, depending on the state to which a decision node belongs.

Case 1. Decision nodes in state 1.

After a history in state 1 where Player 1 has to propose, the proposal $\bar{\theta}^{1}$ is rejected, either by Player 1 in case $\bar{\theta}_{1}^{1}<0$ or by Player 2 in case $\bar{\theta}_{1}^{1} \geq 0$, and leads ultimately to breakdown, or the acceptance of proposal $\bar{\theta}^{2}$, or the acceptance of proposal $\bar{\theta}^{3}$. In all cases, Player 1 receives a payoff of zero. A one-shot deviation to any other proposal is rejected as well, either by Player 1 or by Player 2, and also leads ultimately to a payoff of zero for sure. Such a deviation is therefore not profitable. 
After a history in state 1 where Player 1 has to respond, any proposal $v$ with $v_{1}<0$ is rejected by Player 1, and ultimately leads to a payoff of zero for sure. A one-shot deviation to acceptance leads to the acceptance of $v$ and a negative payoff for Player 1 , or the rejection of $v$ by Player 2 or Player 3 and a payoff of zero for Player 1 . Such a deviation is therefore not profitable. Any proposal $v$ with $v_{1} \geq 0$ is accepted by Player 1 , next rejected by Player 2, and followed by breakdown of the negotiations or acceptance of $(0,1,0)$ in the next period. The payoff for Player 1 is therefore zero. A one-shot deviation to rejection leads ultimately to a payoff of zero for Player 1 as well and is therefore not profitable.

After a history in state 1 where Player 2 has to respond, any proposal $v$ with $v_{2}<\delta$ or $v_{3}<\delta$ is rejected by Player 2, which results in a payoff of $\delta$ for Player 2. A one-shot deviation to acceptance is followed by an acceptance by Player 3 if $v_{3} \geq \delta$ and leads to payoff $v_{2}<\delta$ for Player 2, so is not profitable, and is followed by a rejection by Player 3 if $v_{3}<\delta$, leading to payoff 0 for Player 2, so is not profitable either. Any proposal $v$ with $v_{2} \geq \delta$ and $v_{3} \geq \delta$ is accepted by Player 2, followed by an acceptance by Player 3, and a payoff of $v_{2}$ for Player 2. A one-shot deviation to rejection leads to a payoff of $\delta$ for Player 2 and is therefore not profitable.

After a history in state 1 where Player 3 has to respond, any proposal $v$ with $v_{3}<\delta$ is rejected by Player 3, resulting in a payoff of $\delta$ for Player 3. A one-shot deviation to acceptance is clearly not profitable. Any proposal $v$ with $v_{3} \geq \delta$ is accepted by Player 3 , leading to a payoff of $v_{3}$ for Player 3 . A one-shot deviation to rejection is clearly not profitable.

Case 2. Decision nodes in state 2.

After a history in state 2 where Player 2 has to propose, the proposal $\bar{\theta}^{2}=(0,1,0)$ by Player 2 is accepted by all players, and leads to utility 1 for Player 2. Since Players 1 and 3 reject proposals which give them a negative payoff, there are no profitable one-shot deviations for Player 2. Since a one-shot rejection by any player leads to payoffs $(0, \delta, 0)$, the one-shot deviation property holds for responders.

Case 3. Decision nodes in state 3.

This case is similar to Case 2 .

Proposition 4.3 describes a continuum of SSPEs, parametrized by the proposal $\bar{\theta}^{1}$ by Player 1. For equilibria with $\bar{\theta}_{1}^{1}<0$, the equilibrium payoffs when starting in state 1 are equal to $u(\bar{\theta}, \bar{A} \mid 1)=(0, \delta /(3-\delta), \delta /(3-\delta))$. For equilibria with $\bar{\theta}_{1}^{1} \geq 0$, it holds that $u(\bar{\theta}, \bar{A} \mid 1)=(0, \delta, 0)$. None of the properties, apart from SSPE existence, mentioned in 
Theorem 3.4 are satisfied. All SSPEs have delay. There is a continuum of SSPEs where Player 1 makes an inefficient proposal, and even if Player 1 makes an efficient proposal, it is still rejected by Player 1 or Player 2. Any element of $V \times\{(0,1,0)\} \times\{(0,0,1)\}$ can be a limit equilibrium, so there is no unique limit equilibrium. When starting in state 1 , limit equilibrium utilities are either equal to $(0,1 / 2,1 / 2)$ or $(0,0,1)$. Finally, limit equilibrium proposals are not equal to each other.

\section{Robustness of the Example}

In this section, we examine the robustness of Example 4.1 to perturbations of the transition probabilities. In particular, we will see that the presence of absorbing states is not vital for equilibrium delay.

Indeed, one may object to Example 4.1 that states 2 and 3 are absorbing, no matter what actions the players take, and therefore an analogue of the irreducibility requirement of Assumption B as made for exogenous protocols is not satisfied. The requirement for endogenous protocols of Assumption C, there is a player such that after his rejection he is the next proposer with positive probability, is satisfied. Moreover, the proof of Properties 2 and 3 of Theorem 3.4 does not make use of the irreducibility assumption or the positive probability to make a counterproposal. Such regularity assumptions are only needed for the proof of Properties 4 and 5 of Theorem 3.4.

Still, we would like to argue that Example 4.1 is robust to perturbations in the transition probabilities and that the violations of Properties 2-5 of Theorem 3.4 are not due to the presence of absorbing states. Consider the case where all the transition probabilities are perturbed by some $\varepsilon \in(0,1 / 3)$. We obtain the following example.

Example 5.1 There are three players and three states, $S=N=\{1,2,3\}$. Each player is the proposer in one state and players respond in ascending order, so we have

$$
\begin{aligned}
& \iota(1)=\left(1, \pi^{0}\right), \\
& \iota(2)=\left(2, \pi^{0}\right), \\
& \iota(3)=\left(3, \pi^{0}\right),
\end{aligned}
$$

where $\pi^{0}$ is the identity. Players have to divide a surplus of one unit, $V=\left\{v \in \mathbb{R}^{3} \mid v_{1}+\right.$ $\left.v_{2}+v_{3} \leq 1\right\}$. We perturb the transitions of Example 4.1 by $\varepsilon \in(0,1 / 3)$. In state $s=1$, the transitions depend on the identity of the player who rejects a proposal,

$$
\begin{aligned}
& p^{1}(1)=\left(\frac{1}{3}, \frac{1}{3}, \frac{1}{3}\right), \\
& p^{2}(1)=(\varepsilon, 1-2 \varepsilon, \varepsilon), \\
& p^{3}(1)=(\varepsilon, \varepsilon, 1-2 \varepsilon) .
\end{aligned}
$$


In states $s=2,3$, the transitions are independent of the identity of the rejecting player,

$$
\begin{aligned}
& p^{i}(2)=(\varepsilon, 1-2 \varepsilon, \varepsilon), \quad i \in N, \\
& p^{i}(3)=(\varepsilon, \varepsilon, 1-2 \varepsilon), \quad i \in N .
\end{aligned}
$$

Consider a strategy profile $(\hat{\theta}, \hat{A})$ with proposals

$$
\begin{aligned}
& \hat{\theta}^{1} \in\left\{v \in V \mid v_{1}<0\right\}, \\
& \hat{\theta}^{2}=(0,1-y, y), \\
& \hat{\theta}^{3}=(0, y, 1-y),
\end{aligned}
$$

where

$$
y=\frac{3 \delta \varepsilon}{(3-\delta)(1-\delta+3 \delta \varepsilon)},
$$

and acceptance sets

$$
\begin{array}{rlr}
\hat{A}^{1, s} & =\left\{v \in V \mid v_{1} \geq 0\right\}, & \\
\hat{A}^{2,1} & =\left\{v \in V \mid v_{2} \geq z, v_{3} \geq z\right\}, 1,2,3, \\
\hat{A}^{2,2} & =\left\{v \in V \mid v_{2} \geq z\right\}, & \\
\hat{A}^{2,3} & =\left\{v \in V \mid v_{2} \geq y\right\}, & \\
\hat{A}^{3, s} & =\left\{v \in V \mid v_{3} \geq z\right\}, & \\
\hat{A}^{3,2} & =\left\{v \in V \mid v_{3} \geq y\right\}, &
\end{array}
$$

where

$$
z=\frac{\delta(1-\delta)\left(3-\delta-9 \delta \varepsilon^{2}\right)+\delta \varepsilon(2-\delta)(6 \delta-3)}{(3-\delta)(1-\delta+3 \delta \varepsilon)} .
$$

When players play according to $(\hat{\theta}, \hat{A})$, Player 1 makes a proposal $\hat{\theta}^{1}$ in $V$ with $\hat{\theta}_{1}^{1}<0$ in state 1 , which is rejected by Player 1 himself, and a transition to each of the three states follows with equal probability. In state 2, Player 2 makes a proposal that gives a payoff of 0 to Player 1, gives the reservation payoff $y$ to Player 3, and keeps the remainder of the surplus himself. State 3 is symmetric to state 2, with the roles of Players 2 and 3 reversed. The proposals $\hat{\theta}^{2}$ and $\hat{\theta}^{3}$ in states 2 and 3 are accepted since $1-y>z$, which follows from the fact that

$$
(3-\delta)(1-\delta)+3 \delta \varepsilon(2-\delta)>\delta(1-\delta)(3-\delta-9 \delta \varepsilon)+\delta \varepsilon(2-\delta)(6 \delta-3) .
$$


Proposition 5.2 For every $\varepsilon \in(0,1 / 6)$, there exists $\bar{\delta}<1$ such that for every $\delta \geq \bar{\delta}$ the strategy profile $(\hat{\theta}, \hat{A})$ is an SSPE in Example 5.1 .

Proof. For $s=1,2,3$, we define the equilibrium utilities conditional on state $s, x^{s}=$ $u(\hat{\theta}, \hat{A} \mid s)$. The symmetry of the game and the strategies implies that $x_{2}^{1}=x_{3}^{1}, x_{1}^{2}=x_{1}^{3}$, $x_{2}^{2}=x_{3}^{3}$, and $x_{3}^{2}=x_{2}^{3}$. It holds that $x^{1}=(0, \delta /(3-\delta), \delta /(3-\delta)), x^{2}=\hat{\theta}^{2}$, and $x^{3}=\hat{\theta}^{3}$, where the expression for $x^{1}$ uses the observation that $x_{2}^{1}=(\delta / 3)+(\delta / 3) x_{2}^{1}$.

To show that $(\hat{\theta}, \hat{A})$ is an SSPE, we verify the one-shot deviation property. We consider three cases, depending on the state to which a decision node belongs.

Case 1. Decision nodes in state 1.

Consider a history in state 1 after which Player 3 has to respond. A rejection followed by play according to $(\hat{\theta}, \hat{A})$ leads to a payoff for Player 3 equal to $\delta \varepsilon x_{3}^{1}+\delta \varepsilon x_{3}^{2}+\delta(1-2 \varepsilon) x_{3}^{3}$. A straightforward, but tedious, calculation reveals that this payoff is equal to $z$. Since Player 3 accepts proposals in state 1 if and only if $v_{3} \geq z$, this shows that the one-shot deviation property is satisfied.

Consider a history in state 1 after which Player 2 has to respond to a proposal $v$. Suppose first that $v_{3} \geq z$. A calculation similar to that in the previous paragraph shows that rejection of $v$ by Player 2 yields a payoff of $z$. Acceptance of $v$ by Player 2 leads to the payoff $v_{2}$ since $v$ is also accepted by Player 3. We conclude that accepting the proposal $v$ if and only if $v_{2} \geq z$ does not violate the one-shot deviation principle.

Suppose now that $v_{3}<z$. As before, rejecting $v$ yields Player 2 a payoff of $z$. If Player 2 accepts $v$, it is rejected by Player 3, yielding a continuation utility equal to $\delta \varepsilon x_{2}^{1}+\delta \varepsilon x_{2}^{2}+\delta(1-2 \varepsilon) x_{2}^{3}$. A straightforward, though tedious, calculation reveals the latter expression to be equal to $y$, and it holds that $y<z$ since

$$
z-y=\frac{(1-\delta)(1-3 \varepsilon)(3-\delta+3 \delta \varepsilon)}{(3-\delta)(1-\delta+3 \delta \varepsilon)}>0 .
$$

Hence $v$ should be rejected by Player 2 .

The verification of the one-shot deviation property for Player 1 is trivial for histories where he responds. Consider a history where Player 1 proposes. Player 1 has a profitable one-shot deviation if and only if he can make a proposal that gives more than $z$ to Players 2 and 3 and a positive payoff to himself. We show that $2 z \geq 1$, which implies the absence of such deviations. A straightforward calculation shows that $2 z \geq 1$ if and only if

$$
(1-\delta)\left(-3+7 \delta-2 \delta^{2}-21 \delta \varepsilon+12 \delta^{2} \varepsilon-18 \delta^{2} \varepsilon^{2}\right) \geq 0 .
$$


The second term in the product on the left-hand side of (1), evaluated at $\delta=1$, is equal to $2-9 \varepsilon-18 \varepsilon^{2}$, which is positive for $\varepsilon \in(0,1 / 6)$. It follows that for every $\varepsilon \in(0,1 / 6)$, there exists $\bar{\delta}<1$ such that for every $\delta \geq \bar{\delta}, 2 z \geq 1$.

Case 2. Decision nodes in state 2.

Player 3 accepts a proposal $v$ if and only if $v_{3} \geq y$, where $y$ equals the continuation payoff of Player 3 following his rejection. This shows that the one-shot deviation property is satisfied.

Player 2 accepts a proposal $v$ if and only if $v_{2} \geq z$, where $z$ equals the continuation payoff of Player 2 following his rejection. We observe that acceptance of $v$ yields Player 2 payoff $v_{2}$ if Player 3 accepts as well, and $z$ if Player 3 rejects $v$. This shows that the one-shot deviation property is satisfied.

The verification of the one-shot deviation property for Player 1 is trivial.

Consider a history in state 2 after which Player 2 proposes. Since the proposal $\hat{\theta}^{2}$ of Player 2 gives Players 1 and 3 the least amount they are willing to accept, there is no profitable one-shot deviation for Player 2 which will be accepted by Players 1 and 3 . Consider a one-shot deviation by Player 2 which is rejected by some player. Ultimately, such a proposal leads to breakdown and payoff 0 for Player 2, or an acceptance of $\hat{\theta}^{2}$ and payoff $1-y$ for Player 2 or an acceptance of $\hat{\theta}^{3}$ and Payoff $y$ for Player 2. Since $y<1-y$, the expected payoff for Player 2 is less than $x_{2}^{2}=1-y$, so the deviation is not profitable.

Case 3. Decision nodes in state 3.

By symmetry, the line of argument is the same as in Case 2.

Proposition 5.3 presents an equilibrium which violates Properties 2 and 3 of Theorem 3.4. It also has the remarkable feature that Player 1's equilibrium payoff is equal to zero in every state. This is surprising, since in the framework of Britz, Herings, and Predtetchinski (2012), the bargaining power of a player is proportional to the probability to propose conditional on his own rejection. In the protocol of Example 5.1, this probability is at least $\varepsilon$ for Player 1, irrespective of the state and irrespective of the identity of the rejecting player.

Consider a strategy profile $(\tilde{\theta}, \tilde{A})$ with proposals

$$
\begin{aligned}
& \tilde{\theta}^{1} \in\left\{v \in V \mid v_{1} \geq 0\right\} \\
& \tilde{\theta}^{2}=\left(0,1-y_{3}, y_{3}\right) \\
& \hat{\theta}^{3}=\left(0, y_{2}, 1-y_{2}\right)
\end{aligned}
$$


where

$$
\begin{aligned}
& y_{2}=\frac{\delta \varepsilon+\delta^{2} \varepsilon-3 \delta^{2} \varepsilon^{2}}{1-\delta+2 \delta \varepsilon+\delta^{2} \varepsilon-3 \delta^{2} \varepsilon^{2}} \\
& y_{3}=\frac{\delta \varepsilon}{1-\delta+2 \delta \varepsilon+\delta^{2} \varepsilon-3 \delta^{2} \varepsilon^{2}}
\end{aligned}
$$

and acceptance sets

$$
\begin{array}{rlr}
\tilde{A}^{1, s} & =\left\{v \in V \mid v_{1} \geq 0\right\}, & s=1,2,3, \\
\tilde{A}^{2,1} & =\left\{v \in V \mid v_{2} \geq z_{2}, v_{3} \geq z_{3}\right\}, & \\
\tilde{A}^{2,2} & =\left\{v \in V \mid v_{2} \geq z_{2}\right\}, & \\
\tilde{A}^{2,3} & =\left\{v \in V \mid v_{2} \geq y_{2}\right\}, & s=1,3, \\
\tilde{A}^{3, s} & =\left\{v \in V \mid v_{3} \geq z_{3}\right\}, & \\
\tilde{A}^{3,2} & =\left\{v \in V \mid v_{3} \geq y_{3}\right\}, &
\end{array}
$$

where

$$
\begin{aligned}
z_{2}= & \frac{\delta-\delta^{2}-2 \delta \varepsilon+4 \delta^{2} \varepsilon-3 \delta^{2} \varepsilon^{2}}{1-\delta+2 \delta \varepsilon+\delta^{2} \varepsilon-3 \delta^{2} \varepsilon^{2}} \\
z_{3}= & \frac{\delta-\delta^{2}-2 \delta \varepsilon+3 \delta^{2} \varepsilon}{1-\delta+2 \delta \varepsilon+\delta^{2} \varepsilon-3 \delta^{2} \varepsilon^{2}}
\end{aligned}
$$

When players play according to $(\tilde{\theta}, \tilde{A})$, Player 1 makes a proposal $\tilde{\theta}^{1}$ in $V$ with $\tilde{\theta}_{1}^{1} \geq 0$ in state 1. A straightforward calculation shows that $z_{2}+z_{3}>1$ if and only if

$$
(1-\delta)(2 \delta-6 \delta \varepsilon-1)>0
$$

Therefore it holds that if $\varepsilon<1 / 6$ and $\delta>1 /(2-6 \varepsilon)$, then Player 2 rejects $\tilde{\theta}^{1}$, and a transition to state 2 follows with high probability. In state 2, Player 2 makes a proposal that gives a payoff of 0 to Player 1 , gives the reservation payoff $y_{2}$ to Player 3 , and keeps the remainder of the surplus himself. State 3 is similar, with the roles of Players 2 and 3 reversed. The proposals $\tilde{\theta}^{2}$ and $\tilde{\theta}^{3}$ in states 2 and 3 are accepted since $1-y_{3} \geq z_{2}$ and $1-y_{2} \geq z_{3}$ which follows respectively from

$$
\begin{aligned}
& 1-\delta+\delta \varepsilon+\delta^{2} \varepsilon-3 \delta^{2} \varepsilon^{2}>\delta(1-\delta)+2 \delta^{2} \varepsilon-3 \delta^{2} \varepsilon^{2}>\delta-\delta^{2}-2 \delta \varepsilon+4 \delta^{2} \varepsilon-3 \delta^{2} \varepsilon^{2}, \\
& 1-\delta+\delta \varepsilon>\delta-\delta^{2}-2 \delta \varepsilon+3 \delta^{2} \varepsilon .
\end{aligned}
$$

Proposition 5.3 For every $\varepsilon \in(0,1 / 6)$, there exists $\bar{\delta}<1$ such that for every $\delta \geq \bar{\delta}$ the strategy profile $(\tilde{\theta}, \tilde{A})$ is an SSPE in Example 5.1 . 
Proof. For $s=1,2,3$, we define the equilibrium utilities conditional on state $s, x^{s}=$ $u(\tilde{\theta}, \tilde{A} \mid s)$. We have that $x^{2}=\tilde{\theta}^{2}, x^{3}=\tilde{\theta}^{3}, x_{1}^{1}=0, x_{2}^{1}=\delta \varepsilon x_{2}^{1}+\delta(1-2 \varepsilon) x_{2}^{2}+\delta \varepsilon x_{2}^{3}$, so

$$
x_{2}^{1}=\frac{\delta-\delta^{2}-2 \delta \varepsilon+4 \delta^{2} \varepsilon-3 \delta^{2} \varepsilon^{2}}{1-\delta+2 \delta \varepsilon+\delta^{2} \varepsilon-3 \delta^{2} \varepsilon^{2}}
$$

and $x_{3}^{1}=x_{3}^{2}$, since a rejection by Player 2 in state 1 leads to the same transitions as a rejection by Player 3 in state 2 .

To show that $(\tilde{\theta}, \tilde{A})$ is an SSPE, we verify the one-shot deviation property. We consider three cases, depending on the state to which a decision node belongs.

Case 1. Decision nodes in state 1.

Consider a history in state 1 after which Player 3 has to respond. A rejection followed by play according to $(\tilde{\theta}, \tilde{A})$ leads to a payoff for Player 3 equal to $\delta \varepsilon x_{3}^{1}+\delta \varepsilon x_{3}^{2}+\delta(1-2 \varepsilon) x_{3}^{3}=z_{3}$. Since Player 3 accepts proposals in state 1 if and only if $v_{3} \geq z_{3}$, this shows that the oneshot deviation property is satisfied.

Consider a history in state 1 after which Player 2 has to respond to a proposal $v$. Suppose first that $v_{3} \geq z_{3}$. A calculation similar to that in the previous paragraph shows that rejection of $v$ by Player 2 yields Player 2 a payoff of $z_{2}$. Acceptance yields $v_{2}$ because $v$ is accepted by Player 3. Hence accepting $v$ if and only if $v_{2} \geq z_{2}$ does not violate the one-shot deviation principle.

Suppose now that $v_{3}<z_{3}$. As before, rejecting $v$ by Player 2 gives payoff $z_{2}$. If Player 2 accepts $v$, then it is rejected by Player 3 and yields the continuation payoff of $y_{2}$. It holds that $y_{2}<z_{2}$ since

$$
z_{2}-y_{2}=\frac{\delta(1-\delta)(1-3 \varepsilon)}{1-\delta+2 \delta \varepsilon+\delta^{2} \varepsilon-3 \delta^{2} \varepsilon^{2}}>0 .
$$

Thus rejecting $v$ does not violate the one-shot deviation principle.

The verification of the one-shot deviation property for Player 1 is trivial for histories where he responds. We have already argued that $z_{2}+z_{3}>1$ if $\delta>1 /(2-6 \varepsilon)$. For such values of $\delta$, Player 1 cannot make a profitable one-shot deviation as a proposer.

Case 2. Decision nodes in state 2.

Player 3 accepts a proposal $v$ if and only if $v_{3} \geq y_{3}$, where $y_{3}$ equals the continuation payoff of Player 3 following his rejection. This shows that the one-shot deviation property is satisfied.

Player 2 accepts a proposal $v$ if and only if $v_{2} \geq z_{2}$, where $z_{2}$ equals the continuation payoff of Player 2 following his rejection. We observe that acceptance of $v$ yields Player 2 
payoff $v_{2}$ if Player 3 accepts as well, and $z_{2}$ if Player 3 rejects $v$. This shows that the one-shot deviation property is satisfied.

The verification of the one-shot deviation property for Player 1 is trivial.

Consider a history in state 2 after which Player 2 proposes. Since the proposal $\tilde{\theta}^{2}$ of Player 2 gives Players 1 and 3 the least amount they are willing to accept, there is no profitable one-shot deviation for Player 2 which will be accepted by Players 1 and 3 . Consider a one-shot deviation by Player 2 which is rejected by some player. Ultimately, such a proposal leads to breakdown and payoff 0 for Player 2, or an acceptance of $\tilde{\theta}^{2}$ and payoff $1-y_{3}$ for Player 2 or an acceptance of $\tilde{\theta}^{3}$ and Payoff $y_{2}$ for Player 2. Since it is easily verified that $y_{2}<1-y_{3}$, the expected payoff for Player 2 is less than $x_{2}^{2}=1-y_{3}$, so the deviation is not profitable.

Case 3. Decision nodes in state 3.

Player 3 accepts a proposal $v$ if and only if $v_{3} \geq z_{3}$, where $z_{3}$ equals the continuation payoff of Player 3 following his rejection. This shows that the one-shot deviation property is satisfied.

Player 2 accepts a proposal $v$ if and only if $v_{2} \geq y_{2}$, where $y_{2}$ equals the continuation payoff of Player 2 following his rejection. We observe that acceptance of $v$ yields Player 2 payoff $v_{2}$ if Player 3 accepts as well, and $y_{2}$ if Player 3 rejects $v$. This shows that the one-shot deviation property is satisfied.

The verification of the one-shot deviation property for Player 1 is trivial.

Consider a history in state 3 after which Player 3 proposes. Since the proposal $\tilde{\theta}^{3}$ of Player 3 gives Players 1 and 2 the least amount they are willing to accept, there is no profitable one-shot deviation for Player 3 which will be accepted by Players 1 and 2. Consider a one-shot deviation by Player 3 which is rejected by some player. Ultimately, such a proposal leads to breakdown and payoff 0 for Player 3, or an acceptance of $\tilde{\theta}^{2}$ and payoff $y_{3}$ for Player 3 or an acceptance of $\tilde{\theta}^{3}$ and Payoff $1-y_{2}$ for Player 3 . Since $y_{3}<1-y_{2}$, the expected payoff for Player 3 is less than $x_{3}^{3}=1-y_{2}$, so the deviation is not profitable.

Propositions 5.2 and 5.3 give us a plethora of limit equilibria. From Proposition 5.2 we can see that any point $\left(\theta^{1}, \theta^{2}, \theta^{3}\right)$ where $\theta_{1}^{1}<0$ and $\theta^{2}=\theta^{3}=\left(0, \frac{1}{2}, \frac{1}{2}\right)$ is a limit equilibrium. Similarly, Proposition 5.3 yields limit equilibria of the form $\left(\theta^{1}, \theta^{2}, \theta^{3}\right)$ with $\theta_{1}^{1}>0$ and

$$
\theta^{2}=\theta^{3}=\left(0, \frac{2-3 \varepsilon}{3-3 \varepsilon}, \frac{1}{3-3 \varepsilon}\right) .
$$

Clearly not all players make the same proposals in the limit. The fact that in both 
cases Players 2 and 3 do make the same proposals in the limit can also be deduced from Theorem 3.4. Indeed, since Player 1's proposal is rejected, one can eliminate state 1 and Player 1, and view the resulting system as an exogenous protocol involving Players 2 and 3 only.

\section{Non-existence of SSPEs}

In the example of the previous section, all properties of Theorem 3.4 are violated, with the exception of the existence of an SSPE. In this section, we will present an example where no SSPE exists at all, neither one with immediate agreement, nor one with delay.

Example 6.1 There are three players and three states, $S=N=\{1,2,3\}$. Each player is the proposer in one state and players respond in ascending order, so we have

$$
\begin{aligned}
& \iota(1)=\left(1, \pi^{0}\right), \\
& \iota(2)=\left(2, \pi^{0}\right), \\
& \iota(3)=\left(3, \pi^{0}\right),
\end{aligned}
$$

where $\pi^{0}$ is the identity. Players have to divide a surplus of one unit, $V=\left\{v \in \mathbb{R}^{3} \mid\right.$ $\left.v_{1}+v_{2}+v_{3} \leq 1\right\}$. In state $s=1$, the transitions depend on the identity of the player who rejects a proposal,

$$
\begin{aligned}
& p^{1}(1)=\left(\frac{1}{3}, \frac{1}{3}, \frac{1}{3}\right), \\
& p^{2}(1)=\left(\frac{1}{2}, \frac{1}{2}, 0\right), \\
& p^{3}(1)=\left(\frac{1}{2}, 0, \frac{1}{2}\right) .
\end{aligned}
$$

In states $s=2,3$, the transitions are independent of the identity of the rejecting player,

$$
\begin{aligned}
& p^{i}(2)=(0,1,0), \quad i \in N, \\
& p^{i}(3)=(0,0,1), \quad i \in N .
\end{aligned}
$$

The continuation probability $\delta$ is equal to $3 / 4$.

The only modification in Example 6.1 when compared to Example 4.1 is the possibility to return to state 1 with probability $1 / 2$ after a rejection by either Player 2 or Player 3.

Proposition 6.2 There is no SSPE in Example 6.1. 
Proof. Suppose $(\theta, A)$ is SSPE. It clearly holds that $\theta^{2}=(0,1,0)$ and $\theta^{3}=(0,0,1)$. Now let $x=u(\theta, A \mid 1)$ be the vector of expected payoffs in a subgame starting in state 1 , and $z^{i}$ be the vector of continuation payoffs after a proposal in state 1 is rejected by Player $i$. We have

$$
\begin{aligned}
& z^{1}=\frac{\delta}{3} x+\frac{\delta}{3}(0,1,0)+\frac{\delta}{3}(0,0,1)=\frac{1}{4} x+\left(0, \frac{1}{4}, \frac{1}{4}\right), \\
& z^{2}=\frac{\delta}{2} x+\frac{\delta}{2}(0,1,0)=\frac{3}{8} x+\left(0, \frac{3}{8}, 0\right) . \\
& z^{3}=\frac{\delta}{2} x+\frac{\delta}{2}(0,0,1)=\frac{3}{8} x+\left(0,0, \frac{3}{8}\right) .
\end{aligned}
$$

We distinguish four possible cases.

Case (A) The proposal $\theta^{1}$ is accepted by Players 1,2 , and 3 .

In this case $x=\theta^{1}$. Since all players accept $\theta^{1}$, we have

$$
\begin{aligned}
& x_{1} \geq z_{1}^{1}=\frac{1}{4} x_{1}, \\
& x_{2} \geq z_{2}^{2}=\frac{3}{8} x_{2}+\frac{3}{8} \\
& x_{3} \geq z_{3}^{3}=\frac{3}{8} x_{3}+\frac{3}{8} .
\end{aligned}
$$

This gives the inequalities $x_{1} \geq 0, x_{2} \geq \frac{3}{5}$, and $x_{3} \geq \frac{3}{5}$, whereas at the same time $x_{1}+x_{2}+$ $x_{3}=\theta_{1}+\theta_{2}+\theta_{3} \leq 1$, a contradiction.

Case (B) The proposal $\theta^{1}$ is accepted by Players 1 and 2, and rejected by Player 3 .

Since Player 3 rejects $\theta^{1}$, we have

$$
x=z^{3}=\frac{3}{8} x+\left(0,0, \frac{3}{8}\right),
$$

so $x=z^{3}=\left(0,0, \frac{3}{5}\right)$. Since Player 2 accepts $\theta^{1}$, we have

$$
z_{2}^{3} \geq z_{2}^{2}=\frac{3}{8} x_{2}+\frac{3}{8}
$$

contradicting that $z_{2}^{3}=x_{2}=0$.

Case (C) The proposal $\theta^{1}$ is accepted by Player 1 and rejected by Player 2 .

Since only proposals offering zero payoff to Player 1 are accepted, it holds that $z_{1}^{1}=0$. Since Player 2 rejects $\theta^{1}$, we have

$$
x=z^{2}=\frac{3}{8} x+\left(0, \frac{3}{8}, 0\right)
$$

so $x=z^{2}=\left(0, \frac{3}{5}, 0\right)$. Therefore,

$$
z^{3}=\frac{3}{8} x+\left(0,0, \frac{3}{8}\right)=\left(0, \frac{9}{40}, \frac{3}{8}\right) .
$$


Suppose Player 1 makes the proposal

$$
v=\left(\frac{1}{120}, \frac{3}{5}+\frac{1}{120}, \frac{3}{8}+\frac{1}{120}\right) .
$$

Since $v_{3}>z_{3}^{3}$ the proposal $v$ is accepted by Player 3. Since $v_{2}>z_{2}^{2}$ it is accepted by Player 2 also. And since $v_{1}>z_{1}^{1}=0$ the proposal $v$ is accepted by Player 1 . But then proposing $v$ is a profitable one-shot deviation for Player 1.

Case (D) The proposal $\theta^{1}$ is rejected by Player 1.

If Player 1 rejects his own proposal, then $x=z^{1}$, so $x=\left(0, \frac{1}{3}, \frac{1}{3}\right)$. It now follows by exactly the same argument as in Case (C) that Player 1 has a profitable one-shot deviation.

Perturbing the transition functions slightly will not affect the conclusion of the proposition. When we parametrize games by the transition functions, one can show that the set of games having an SSPE is closed.

\section{Conclusion}

We consider bargaining games of perfect information with a unanimous acceptance rule. The focus of the analysis is on the selection of the proposer. In the model considered, the proposer and the order of the responding players is determined by the state. The probability distribution over states in the following period is determined jointly by the current state and the identity of the player who rejected the previous proposal.

Britz, Herings, and Predtetchinski (2010, 2012) study two extreme special cases of this framework: one where the identity of the future proposer only depends on the identity of the current proposer, and one where it only depends on the identity of the rejector. In both cases it is shown that subgame perfect equilibrium in pure stationary strategies exist, are efficient, and have the immediate acceptance property. Asymptotically, as players become perfectly patient, all such equilibria converge to the appropriately defined asymmetric Nash bargaining solution.

In this paper, however, we show that these conclusions do not carry over to the general framework: subgame perfect equilibria in pure stationary strategies need not exist. When they do exist, such equilibria may exhibit delay and may be inefficient. The limit equilibrium is not necessarily unique.

One message in Britz, Herings, and Predtetchinski (2012) is that the bargaining power of a player is determined by the probability to propose conditional on that player's rejection. 
One of our examples shows that even though players can propose with positive probability conditional on their rejection in every state, they might still have no bargaining power at all.

\section{References}

Ausubel, L.M., P. Cramton, And R.J. Deneckere (2002), "Bargaining with Incomplete Information," in R.J. Aumann and S. Hart (eds.), Handbook of Game Theory, Volume 3, Elsevier Science Publishers, Amsterdam, pp. 1897-1945.

Banks, J., And J. Duggan (2000), "A Bargaining Model of Collective Choice," American Political Science Review, 94, 73-88.

Binmore, K., A. Rubinstein, And A. Wolinsky (1986), "The Nash Bargaining Solution in Economic Modelling," RAND Journal of Economics, 17, 176-188.

Bloch, F. (1996), "Sequential Formation of Coalitions in Games with Externalities and Fixed Payoff Division," Games and Economic Behavior, 14, 90-123.

Britz, V., P.J.J. Herings, And A. Predtetchinski (2010), "Non-cooperative Support for the Asymmetric Nash Bargaining Solution," Journal of Economic Theory, 145, 1951-1967.

Britz, V., P.J.J. Herings, And A. Predtetchinski (2012), "On the Convergence to the Nash Bargaining Solution for Endogenous Protocols," Meteor Research Memorandum RM/12/030, Maastricht University, forthcoming in Games and Economic Behavior.

Chatterjee, K., B. Dutta, D. Ray, and K. Sengupta (1993), "A Noncooperative Theory of Coalitional Bargaining," Review of Economic Studies, 60, 463-477.

Fudenberg, D., And J. Tirole (1991), Game Theory, MiT Press, Cambridge, Massachusetts.

Haller, H. (1986), "Non-cooperative Bargaining of $N \geq 3$ Players," Economics Letters, 22, 11-13.

Hart, S., ANd A. Mas-Colell (1996), "Bargaining and Value," Econometrica, 64, 357-380.

Herings, P.J.J., And A. Predtetchinski (2009), "Bargaining with Non-convexities," METEOR Research Memorandum 09/42, Maastricht University, Maastricht, pp. 1-16.

Herrero, M.J. (1985), A Strategic Bargaining Approach to Market Institutions, PhD Thesis, London School of Economics, 1-111.

Jéhiel, P. And B. Moldovanu (1995), "Cyclical Delay in Bargaining with Externalities," The Review of Economic Studies, 62, 619-637.

Kalandrakis, T. (2004), "Equilibria in Sequential Bargaining Games as Solutions to Systems of Equations," Economics Letters, 84, 407-411.

KaWAmori, T. (2008), "A Note on Selection of Proposers in Coalitional Bargaining," International Journal of Game Theory, 37, 525-532. 
Kultti, K., AND H. Vartiainen (2010), "Multilateral Non-Cooperative Bargaining in a General Utility Space," International Journal of Game Theory, 39, 677-689.

Laruelle, A., And F. Valenciano (2008), "Noncooperative Foundations of Bargaining Power in Committees and the Shapley-Shubik Index," Games and Economic Behavior, 63, 341-353.

Maskin, E.S., And J. Tirole (2001), "Markov Perfect Equilibrium, I. Observable Actions," Journal of Economic Theory, 100, 191-219.

Merlo, A., And C. Wilson (1995), "A Stochastic Model of Sequential Bargaining with Complete Information," Econometrica, 63, 371-399.

Miyakawa, T. (2008), "Noncooperative Foundation of $n$-Person Asymmetric Nash Bargaining Solution," Journal of Economics of Kwansei Gakuin University, 62, 1-18.

Laruelle, A., And F. Valenciano (2008), "Noncooperative Foundations of Bargaining Power in Committees and the Shapley-Shubik Index," Games and Economic Behavior, 63, 341-353.

Rubinstein, A. (1982), "Perfect Equilibrium in a Bargaining Model," Econometrica, 50, 97-109.

Selten, R. (1981), "A Noncooperative Model of Characteristic Function Bargaining," in V. Böhm and H.H. Nachtkamp (eds.), Essays in Game Theory and Mathematical Economics in Honor of Oskar Morgenstern, Bibliografisches Institut Mannheim, pp. 131-151. 